Ultra-fast photoluminescence in fused silica surface flaws susceptible to laser damage

T. A. Laurence, J. D. Bude, N. Shen

May 9, 2011

Materials Research Society Spring Meeting San Francisco, CA, United States April 25, 2011 through April 29, 2011 
This document was prepared as an account of work sponsored by an agency of the United States government. Neither the United States government nor Lawrence Livermore National Security, LLC, nor any of their employees makes any warranty, expressed or implied, or assumes any legal liability or responsibility for the accuracy, completeness, or usefulness of any information, apparatus, product, or process disclosed, or represents that its use would not infringe privately owned rights. Reference herein to any specific commercial product, process, or service by trade name, trademark, manufacturer, or otherwise does not necessarily constitute or imply its endorsement, recommendation, or favoring by the United States government or Lawrence Livermore National Security, LLC. The views and opinions of authors expressed herein do not necessarily state or reflect those of the United States government or Lawrence Livermore National Security, LLC, and shall not be used for advertising or product endorsement purposes. 


\title{
Ultra-fast photoluminescence in fused silica surface flaws susceptible to laser damage
}

\author{
Ted A. Laurence ${ }^{1}$, Jeff D. Bude ${ }^{1}$, and Nan Shen ${ }^{1}$ \\ ${ }^{1}$ Condensed Matter and Materials Division, Lawrence Livermore National Laboratory \\ Livermore, CA 94550, U.S.A.
}

\begin{abstract}
Using high-sensitivity confocal time-resolved photoluminescence (PL) techniques, we found an ultrafast PL (40 ps-5 ns) from impurity-free surface flaws on fused silica. This PL is excited by the single-photon absorption of sub-band gap light. Regions which exhibit this PL are strongly absorptive well below the band gap, as evidenced by a propensity to damage with 3.5 $\mathrm{eV}$ nanosecond-scale laser pulses. Very high defect densities are needed to explain the damage thresholds observed. For such high defect densities, significant interactions between defects may strongly affect the temporal characteristics of the emission of electronic excitations. We propose that the distribution in lifetimes observed is not simply due to a large variety of defect states, but due to a variety of energy transfer interactions between defect states.
\end{abstract}

\section{INTRODUCTION}

The goal of the National Ignition Facility at Lawrence Livermore National Laboratory is to obtain nuclear fusion in a laboratory setting using laser-based inertial confinement fusion. Laser pulses from 192 beams implode a fuel capsule, increasing the temperature and pressure to the regime where nuclear fusion can occur. Such high energy laser pulses can cause damage in the final focusing optics. This laser damage from nanosecond-scale pulses occurs in a regime with important differences from femtosecond laser damage. For nanosecond scale pulses, the electronic excitations have sufficient time to transfer their energy to the lattice [1]. This fact plays a key role in the development of laser-assisted absorption fronts [2], which lead to the large damage sites caused by temperature-activated absorption. Another important point is that, for the ns-scale pulses at the focusing optics, the intensities in pulses for which damage occurs is only near $2 \mathrm{GW} / \mathrm{cm}^{2}$, which is much lower than intensities at which multiphoton absorption $\chi^{(3)}$ processes are observed. In order to explain this laser damage, high defect densities are required. We previously estimated that absorptivities of at least $\alpha=1000 \mathrm{~cm}^{-1}$ are required to explain laser damage of silica surface flaws [3]. For example, if the absorption cross-section $\sigma$ of a defect is very high at $10^{-16} \mathrm{~cm}^{-2}$, the defect density required is $n=\alpha / \sigma=10^{19} \mathrm{~cm}^{-3}$. For smaller crosssections, $n$ would have to be greater.

We recently discovered a novel photoluminescence with distributed fluorescence lifetimes in surface flaws in fused silica that are associated with laser damage by high fluence, ns-scale lasers [3]. This PL exhibits a broad distribution in lifetimes and emission and excitation spectra; some of the spectral characteristics of this PL were observed previously [4]. We have only found it associated with surfaces and nano-structured materials, particularly surfaces created by fractures [5]. The surfaces from which we observe this PL have a high density of defects. At such high defect densities, interactions between the defects likely become important. We suggest that Förster Resonance Energy Transfer (FRET) [6, 7] and other energy transfer mechanisms lead to the broad distribution in lifetimes observed. In particular, energy transfer between randomly distributed donors (also called sensitizers) and acceptors leads to stretched 
exponential decay models [8-10]. We develop a way to fit such models, accounting for instrument response and low photon count histograms. We find that the model fits our data well, suggesting that FRET may play a role in the novel photoluminescence in laser damage-prone fused silica.

\section{THEORY AND EXPERIMENT}

\section{$\underline{\text { Stretched exponential decay model }}$}

The high defect densities required to explain nanosecond-scale laser damage lead to small average spacing between defects. For the value mentioned above of $n=10^{19} \mathrm{~cm}^{-3}$, the average distance is just under $5 \mathrm{~nm}$. For higher densities obtained for smaller absorption cross-sections, distances between defects will be smaller. At these distances, energy transfer via dipole-dipole interactions may affect the temporal characteristics of the fluorescence lifetime decays.

The expected fluorescence lifetime decay of a set of donor defects in the presence of a randomly distributed acceptor species has been extensively studied. The model was first worked out in the three-dimensional case by Foerster [9]. Additional cases have been worked out subsequently [8]. The generalization to fractal dimensions was found in Klafter et al. [10]. In each case, a stretched exponential model was obtained.

Consider an energy transfer transfer rate between a donor and acceptor of the form

$$
\omega(R)=\frac{1}{\tau}\left(\frac{R_{0}}{R}\right)^{s}
$$

The rate of emission from a donor species in the presence of an acceptor species with concentration $n$ is [8]:

$$
k(t)=\exp \left[-V_{\Delta} n R_{0}^{\Delta} \Gamma(1-\Delta / s)(t / \tau)^{\Delta / s}\right]
$$

$V_{\Delta}$ is the volume of unit sphere in $\Delta$ dimensions. This expression may also be used for fractal dimensions $\Delta[10]$.

By combining many of these parameters, a simplified fitting model may be used:

$$
P(t)=A \exp \left[-(t / \tau)^{\beta}\right]
$$

For using this model to fit experimental data obtained via time-correlated single photon counting (TCSPC) [11-13], it is necessary for the model to account for the finite bin width of the measurements, and the instrument response. Wraparound of the fluorescence decay due to the high repetition rate of the lasers used for TCSPC must also be taken into account. If the duration of each time bin is $\Delta t$, and the time to the next excitation pulse is $N \Delta t$, then integrating Eq. (0.3) we obtain for the $i^{\text {th }}$ bin

$$
P_{i}=\sum_{j=0}^{\infty} \frac{1}{\Gamma\left(\beta^{-1}\right)}\left\{\Gamma\left[\beta^{-1},\left(\frac{(j N+i+1) \Delta t}{\tau}\right)^{\beta}\right]-\Gamma\left[\beta^{-1},\left(\frac{(j N+i) \Delta t}{\tau}\right)^{\beta}\right]\right\}
$$


$\Gamma(a, x)$ is the incomplete gamma function, and $\Gamma(a)$ is the gamma function. The functional form of $(0.4)$ is then convolved with a measured instrument response $\left(\operatorname{IRF}_{i}\right)$, and fitted to the data using a maximum likelihood procedure as described in [12]. One additional fitting parameter in addition to $A$, $\tau$, and $\beta$ is a temporal shift in the position of the IRF.

\section{Experiment}

The fluorescence lifetime decays of fused silica surface flaws are measured as described previously [3]. Briefly, a $405 \mathrm{~nm}$ pulsed laser diode with 150 ps pulse widths (LDH-405, Picoquant, Berlin, Germany) was focused using a high numerical aperture objective onto a $2 \mathrm{~N}$ Vicker's indentation. The fluorescence was collected using the same objective, filtered (using a $580 \mathrm{~nm}$ bandpass filter), and measured using a single photon counting avalanche photodiode (PDM module, Micro Photon Devices, Bolzano, Italy). The time difference between the laser pulses and the fluorescence photons is measured using a PicoHarp 300 (Picoquant, Berlin, Germany). The instrument response is measured by exciting Silver nanoparticles dried on a fused silica substrate.

\section{RESULTS}

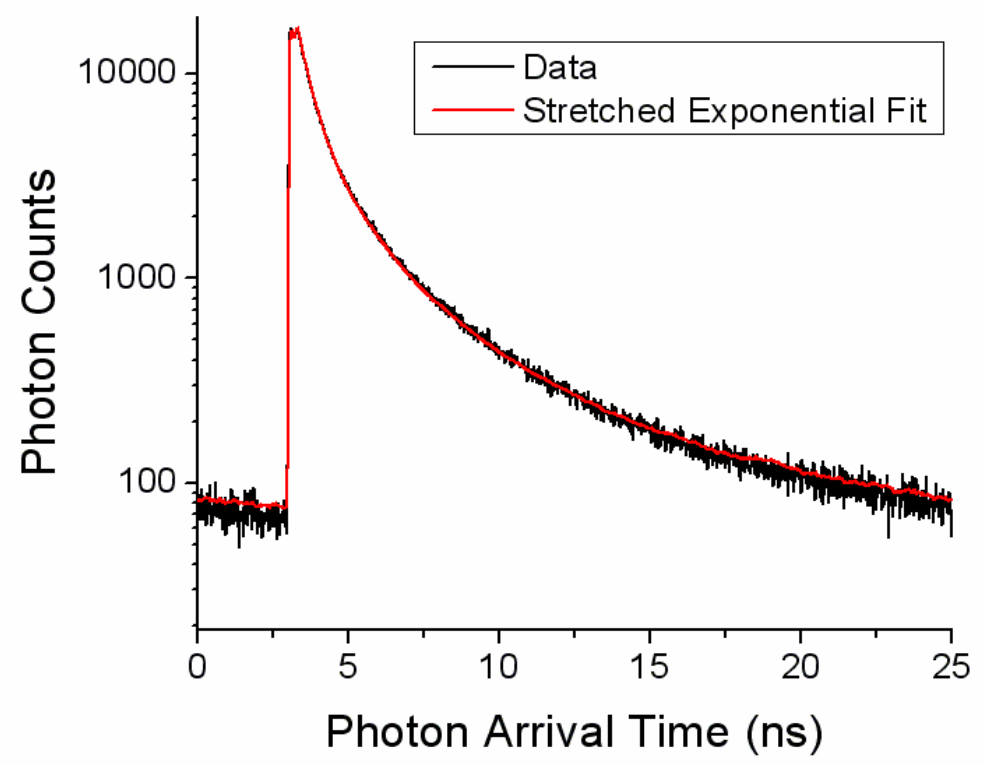

Figure 1: Fluorescence lifetime decay of $2 \mathrm{~N}$ silica Vicker's indentation is fitted to stretched exponential fit, accounting for instrument response and finite bin widths.

In Figure 1, we show the fluorescence lifetime curve measured from a 2N Vicker's indentation on fused silica. The stretched exponential model developed in the previous section fits well to the fluorescence lifetime decay. The quality of fit obtained with the four parameters of the stretched exponential fit (A, $\tau, \beta$, and IRF shift; $\left.\chi^{2}=1.6\right)$ is comparable to that obtained by a four-exponential fit with varying lifetimes (10 parameters; $\left.\chi^{2}=1.2\right)$ and a four-exponential fit with fixed lifetimes of $0.04,0.2,1.0$, and $5 \mathrm{~ns}\left(6\right.$ parameters; $\left.\chi^{2}=2.0\right)$. The normalized residuals calculated from the stretched exponential fit are similar to those of the other fits (Figure 2). The 
fit is good over two orders of magnitude in photon arrival time. The high quality fit of the stretched exponential with fewer parameters is consistent with the model described above where energy transfer interactions play an important role in the temporal characteristics of the fluorescence lifetime decay of the novel photoluminescence or fluorescence from fused silica surface flaws.

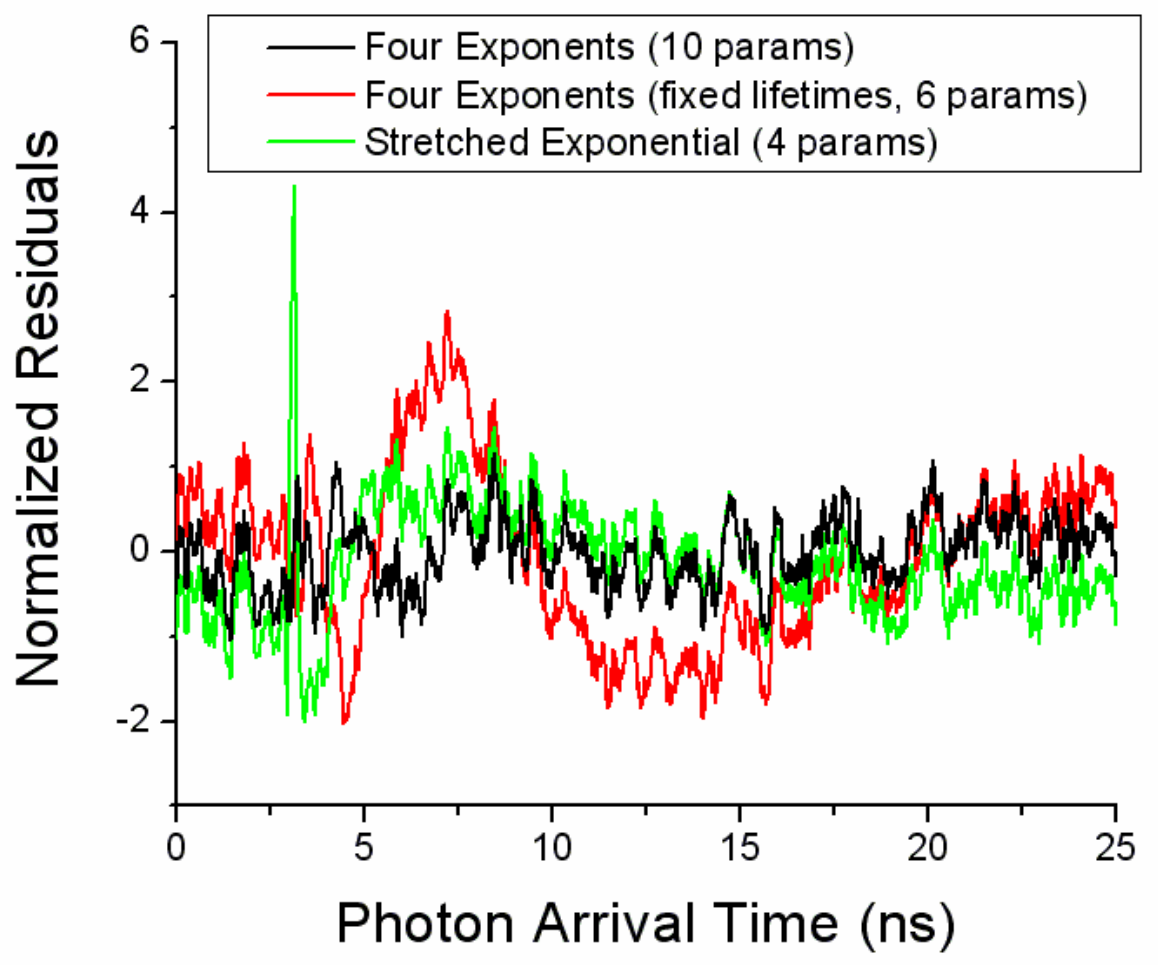

Figure 2: Normalized residuals plotted for three different fits of the data in Figure 1. The fits include a four-exponential fit with 10 parameters, a four-exponential fit with fixed lifetimes and 6 parameters, and the stretched exponential fit with 4 parameters.

\section{DISCUSSION AND CONCLUSION}

We have proposed that the distributed fluorescence lifetimes measured in fused silica surface flaws are related energy transfer interactions within a high density of defects. The stretched exponential model naturally results from these models, and is shown to fit the data well with fewer parameters than other common decay models. These results offer preliminary support for this model. Further experiments detailing spectral characteristics and varying concentrations of defects will shed further light on the role of energy transfer interactions.

\section{ACKNOWLEDGMENTS}


This work performed under the auspices of the U.S. Department of Energy by Lawrence Livermore National Laboratory under Contract DE-AC52-07NA27344. We thank Rusty Steele for assistance in the preparation of the fused silica samples.

\section{REFERENCES}

1. Begin typing text here.

1. Papernov, S. and A.W. Schmid. Laser-induced surface damage of optical materials: absorption sources, initiation, growth, and mitigation. in Laser-Induced Damage in Optical Materials: 2008. 2008. Boulder, CO, USA: SPIE.

2. Carr, C.W., J.D. Bude, and P. DeMange, Laser-supported solid-state absorption fronts in silica. Physical Review B (Condensed Matter and Materials Physics), 2010. 82(18): p. 184304.

3. Laurence, T.A., J.D. Bude, N. Shen, T. Feldman, P.E. Miller, W.A. Steele, and T. Suratwala, Metallic-like photoluminescence and absorption in fused silica surface flaws. Applied Physics Letters, 2009. 94(15): p. 151114.

4. Kucheyev, S.O. and S.G. Demos, Optical defects produced in fused silica during laserinduced breakdown. Applied Physics Letters, 2003. 82(19): p. 3230-3232.

5. Miller, P.E., J.D. Bude, T.I. Suratwala, N. Shen, T.A. Laurence, W.A. Steele, J. Menapace, M.D. Feit, and L.L. Wong, Fracture-induced subbandgap absorption as a precursor to optical damage on fused silica surfaces. Opt. Lett., 2010. 35(16): p. 2702.

6. Dexter, D.L., A Theory of Sensitized Luminescence in Solids. Journal of Chemical Physics, 1953. 21(5): p. 836-850.

7. Forster, T., Energiewanderung und Fluoreszenz. Die Naturwissenschaften, 1946. 6: p. 166-175.

8. Blumen, A., Excitation Transfer From A Donor To Acceptors In Condensed Media - A Unified Approach. Nuovo Cimento Della Societa Italiana Di Fisica B-General Physics Relativity Astronomy And Mathematical Physics And Methods, 1981. 63(1): p. 50-58.

9. Forster, T., Experimentelle Und Theoretische Untersuchung Des Zwischenmolekularen Ubergangs Von Elektronenanregungsenergie. Zeitschrift Fur Naturforschung Section AA Journal Of Physical Sciences, 1949. 4(5): p. 321-327.

10. Klafter, J. and A. Blumen, Fractal Behavior In Trapping And Reaction. Journal Of Chemical Physics, 1984. 80(2): p. 875-877.

11. Becker, W., Advanced Time-Correlated Single Photon Counting Techniques. Springer Series in Chemical Physics, ed. J.P.T.a.W.Z. A. W. Castleman. Vol. 81. 2005, Heidelberg, New York: Springer.

12. Laurence, T.A. and B.A. Chromy, Efficient maximum likelihood estimator fitting of histograms. Nature Methods, 2010. 7(5): p. 338-339.

13. Zander, C., K.H. Drexhage, K.T. Han, J. Wolfrum, and M. Sauer, Single-molecule counting and identification in a microcapillary. Chemical Physics Letters, 1998. 286(56): p. 457-65. 